\title{
Beating the Rayleigh Limit Using Two-Photon Interference
}

\author{
Michał Parniak, ${ }^{1,2, *}$ Sebastian Borówka, ${ }^{1}$ Kajetan Boroszko, ${ }^{1}$ Wojciech Wasilewski, ${ }^{1,2}$ \\ Konrad Banaszek, ${ }^{1,2}$ and Rafał Demkowicz-Dobrzański ${ }^{1}$ \\ ${ }^{1}$ Faculty of Physics, University of Warsaw, Pasteura 5, 02-093 Warsaw, Poland \\ ${ }^{2}$ Centre for Quantum Optical Technologies, Centre of New Technologies, University of Warsaw, Banacha 2c, 02-097 Warsaw, Poland
}

(Received 7 August 2018; revised manuscript received 3 October 2018; published 18 December 2018)

\begin{abstract}
Multiparameter estimation theory offers a general framework to explore imaging techniques beyond the Rayleigh limit. While optimal measurements of single parameters characterizing a composite light source are now well understood, simultaneous determination of multiple parameters poses a much greater challenge that in general requires implementation of collective measurements. Here we show, theoretically and experimentally, that Hong-Ou-Mandel interference followed by spatially resolved detection of photons provides precise information on both the separation and the centroid for a pair of point emitters, avoiding trade-offs inherent to single-photon measurements.
\end{abstract}

DOI: 10.1103/PhysRevLett.121.250503

Multiparameter quantum estimation emerges as a general framework to optimize information retrieval in a variety of experimental scenarios. The problem of imaging can be viewed as an important example of such a scenario, where the properties of an image, for example, locations and intensities of point emitters, or the moments of the image intensity distribution are the parameters to be estimated [1-7]. A recently introduced family of superresolution imaging schemes [8-13] based on spatial demultiplexing enables one to determine the separation of two nearby point sources below the Rayleigh limit, but requires in principle perfect knowledge of the centroid [4]. Moreover, at the single-photon level they are fundamentally incompatible with the measurement needed to estimate the centroid itself. Nonetheless, the effort to extract optimally information carried in light emitted naturally by a source [13-19] may open up new applications compared to established approaches that require manipulations of the sample to be imaged [20].

A deeper insight rooted in the multiparameter estimation theory reveals a possible solution of the above incompatibility problem. Interestingly, in the strong subdiffraction regime where images of the sources overlap significantly, the problem can be modeled as simultaneous estimation of the length and the rotation angle of a qubit Bloch vector [4]. From the theory of multiparameter estimation it then follows that, provided collective measurement on the photons (or qubits) is allowed, the incompatibility between

Published by the American Physical Society under the terms of the Creative Commons Attribution 4.0 International license. Further distribution of this work must maintain attribution to the author(s) and the published article's title, journal citation, and DOI. the optimal individual measurements to estimate the centroid and the sources' separation ceases to be an issue $[21,22]$. The question is how to realize such a collective measurement in practice.

In this Letter, we exploit the advantages offered by the multiphoton interference approach, demonstrating a twophoton (2P) protocol for imaging of two point sources, where the centroid estimation is performed in the optimal way, and at the same time the sources' separation parameter is estimated with a superresolution precision. The idea relies on the effect of two-photon interference and does not require preestimation of the centroid or fine-tuning of the measurement basis inherent to spatial mode demultiplexing schemes [8-13], where any systematic error in the centroid estimation propagates to the separation estimation and significantly degrades the imaging protocol.

In Fig. 1(a) we depict a scenario where two photons emitted by a composite source arrive simultaneously at the input ports of the beam splitter. The proposed protocol exploits both cross-coincidences between the output ports and double events in each port, detected with spatial resolution [23]. The number of cross-coincidences grows with the distinguishability of the two photons and therefore carries information about the separation between point sources. Most importantly, the proposed interferometric scheme does not require prior selection of the measurement basis or the axis of symmetry, as the two photons serve as a reference for each other. Furthermore, thanks to spatially resolved detection this strategy will be shown to be robust against residual spectral distinguishability. Let us note that previous approaches to collective measurements relied on the fundamental advantage of using photonic entanglement [24], also for superresolution photolithography [25-28], which is essentially different from our technique of simply utilizing the bosonic nature of photons. 
(a)

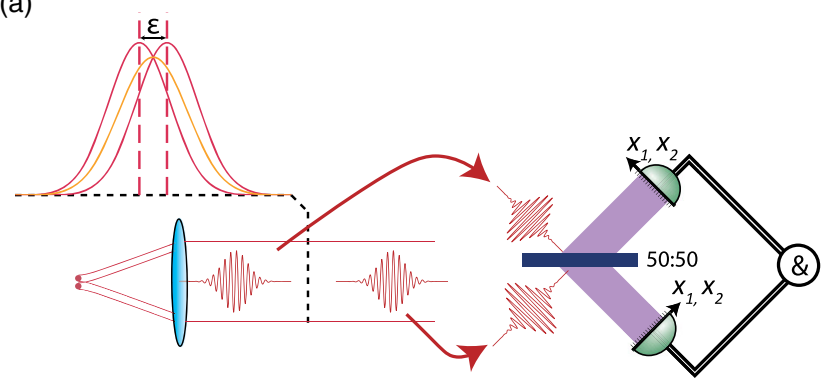

(b)

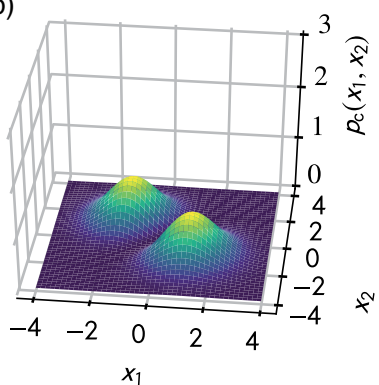

(c)

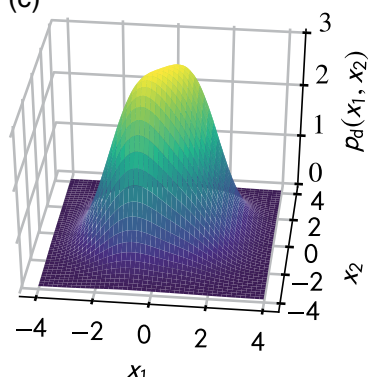

FIG. 1. The idea for collectively enhanced quantum imaging protocol. (a) Two point sources are imaged using an optical system with a well-defined intensity point-spread function (inset: all curves are normalized to equal integrals). The photons are made to interfere (see text for details) at the output beam splitter, after which we register cross-coincidences and double events with spatial resolution. Information about sources' separation $\varepsilon$ as well as centroid $x_{0}$ are drawn both from the ratio of cross-coincidences (b) to double events (c) and their spatial probability distributions $p_{c}\left(x_{1}, x_{2}\right)$ and $p_{d}\left(x_{1}, x_{2}\right)$ (here expressed in arbitrary units).

The somewhat nontrivial demand of interfering two photons from a realistic classical (thermal) composite source on a beam splitter could be realized by a photon number quantum nondemolition (QND) measuring device that preserves spatial properties of light, and upon registering single photons delays and redirects them so that they arrive together at the two beam splitter input ports. Recent advances in storing and controlling single photons in quantum nonlinear media such as Rydberg atoms [29] as well as spatially multimode quantum memories [30] with processing capabilities [31] could provide a viable way to realize the scheme. In particular, a $\pi$ phase shift induced by a single photon has already been achieved [32] and current experiments already explore the Rydberg interactions in the transverse spatial domain [33]. The combination of a multimode quantum memory with the spatially resolving QND measurement could follow the steps of experiments demonstrating optical storage in Rydberg media [34,35], use alternative proposals such as nonlinearities induced by acStark shifts [36], or utilize novel solid-state systems with similar capabilities yet broader spectral bandwidths [37,38].

To support the intuitions behind the discussed scheme, let us compare the two-photon imaging scheme with direct imaging (DI) by modeling a problem of resolving a 1D image formed by two point sources. Let $\psi\left(x-x_{0}\right)$ be a 1D

wave function representing the amplitude transfer function of a single source in the image plane centered at point $x_{0}$. We assume that this function is determined by wellcharacterized properties of the imaging setup. In what follows we denote the corresponding single-photon state characterized by $\psi\left(x-x_{0}\right)$ as $\left|x_{0}\right\rangle$.

Consider a situation where the image is produced as a result of an incoherent overlap of images of two point sources separated by a distance $\varepsilon$, located at $x_{+}=x_{0}+\varepsilon / 2$ and $x_{-}=x_{0}-\varepsilon / 2$. We may then write the spatial density matrix of a photon emitted from the system as $\rho=1 / 2\left(\left|x_{+}\right\rangle\left\langle x_{+}|+| x_{-}\right\rangle\left\langle x_{-}\right|\right)$.

In the DI scheme the probability distribution for the position of the detected photon is given by $p_{\boldsymbol{\theta}}(x)=\frac{1}{2}\left|\psi\left(x-x_{+}\right)\right|^{2}+\frac{1}{2}\left|\psi\left(x-x_{-}\right)\right|^{2}$, where $\boldsymbol{\theta}=\left[\left(x_{+}+x_{-}\right) /\right.$ $\left.2, x_{+}-x_{-}\right]=\left(x_{0}, \varepsilon\right)$ represents the dependence on the estimated parameters. For any locally unbiased estimator, the covariance matrix for the estimated parameters can be lower bounded using the Cramér-Rao inequality [39]:

$$
\operatorname{Cov} \boldsymbol{\theta} \geq \frac{F^{-1}}{N}, \quad F_{i j}=\int_{-\infty}^{\infty} d x \frac{\partial_{\theta_{i}} p_{\boldsymbol{\theta}}(x) \partial_{\theta_{j}} p_{\boldsymbol{\theta}}(x)}{p_{\boldsymbol{\theta}}(x)},
$$

where $F_{i j}$ is the Fisher information (FI) matrix per single photon, while $N$ represents the total number of photons registered. The bound is asymptotically saturable using, e.g., a max-likelihood estimator; hence, $\lim _{N \rightarrow \infty} N \operatorname{Cov} \boldsymbol{\theta}=F^{-1}$. As the FI matrix is diagonal for the given problem, we can easily calculate the variances $\Delta^{2} x_{0}=\left(F^{-1}\right)_{11}, \Delta^{2} \varepsilon=$ $\left(F^{-1}\right)_{22}$ of the estimated parameters per single photon used. In the case of DI, the FI matrix yields the following precision for estimation in the leading order in $\varepsilon$ :

$$
\begin{gathered}
\left(\Delta^{2} x_{0}\right)_{\mathrm{DI}}^{-1}=1-\frac{\varepsilon^{2}}{4}, \\
\left(\Delta^{2} \varepsilon\right)_{\mathrm{DI}}^{-1}=\frac{\varepsilon^{2}}{8}
\end{gathered}
$$

where for concreteness we have assumed a Gaussian-shaped transfer function $\psi(x)=(2 \pi)^{-1 / 4} \exp \left(-x^{2} / 4\right)$, yielding an intensity profile with standard deviation 1 , which can be regarded as a natural unit of distance in the problem. The above expansion is valid for small $\varepsilon$ when source point images are separated by a distance smaller than the transfer function spread, and clearly shows impossibility of precise estimation of $\varepsilon$ in the $\varepsilon \rightarrow 0$ limit.

Crucially, as observed in Ref. [10], a more fundamental bound based on the quantum FI matrix $F^{Q}$ [40], which does not assume any particular measurement strategy and is based solely on the properties of the quantum state $\rho$ to be measured, reads

$$
\left(\Delta^{2} x_{0}\right)_{Q}^{-1}=1-\frac{\varepsilon^{2}}{4}
$$




$$
\left(\Delta^{2} \varepsilon\right)_{Q}^{-1}=\frac{1}{4}
$$

indicating a potential spectacular robustness of the $\varepsilon$ estimation as the $\Delta^{2} \varepsilon$ is constant irrespectively of how small $\varepsilon$ is. While the bound Eq. (1) with $F$ being replaced by $F^{Q}$ is saturable for the problem considered, it requires collective measurements on many copies of $\rho[1,4,5,22]$.

We are now ready to quantify the precision of estimating $x_{0}$ and $\varepsilon$ in the two-photon interferometric scheme and contrast it with the abovementioned strategies. Given $\rho^{\otimes 2}$ at the input ports of the beam splitter, we calculate spatially resolved probabilities for coincidences $p_{c}\left(x_{1}, x_{2}\right)$ as well as double events $p_{d}\left(x_{1}, x_{2}\right)$, from which the information about $x_{0}$ and $\varepsilon$ is drawn. Furthermore, we assume a known two-photon visibility $\mathcal{V}$ resulting from the operation of the nondemolition photon routing device before the beam splitter. The resulting precision of estimation per single photon used, see Supplemental Material [41], expanded up to the second order in $\varepsilon$ reads

$$
\begin{gathered}
\left(\Delta^{2} x_{0}\right)_{2 \mathrm{P}}^{-1}=1-\frac{\varepsilon^{2}}{4}, \\
\left(\Delta^{2} \varepsilon\right)_{2 \mathrm{P}}^{-1}= \begin{cases}\frac{1}{8}+\frac{5}{128} \varepsilon^{2} & \mathcal{V}=1 \\
\frac{4-\mathcal{V}^{2}}{32\left(1-\mathcal{V}^{2}\right)} \varepsilon^{2} & \mathcal{V}<1,\end{cases}
\end{gathered}
$$

while the expansion in case of imperfect visibility is valid in the regime where $\varepsilon^{2} \lesssim 1-\mathcal{V}$. In case of perfect interference, we see that while keeping the optimality of the $x_{0}$ estimation, we additionally obtain the $\varepsilon$ estimation with precision reduced by approximately a factor of 2 compared to the fundamental bound given in Eq. (5). This shows superiority of $2 \mathrm{P}$ over DI, with the added advantage that the measurement setting is fixed and does not require adjusting the measurement for $\varepsilon$ depending on preestimation of $x_{0}$. Here we would like to stress the importance of spatial information that is available in the experiment: if only the ratio of coincidence and double events was available, there would be no information on the $x_{0}$ parameter at all, while the precision of the $\varepsilon$ estimation shows a small reduction for finite $\varepsilon$ compared with Eq. (7) and reads as $\frac{1}{8}-\frac{5}{128} \varepsilon^{2}+$ $O\left(\varepsilon^{4}\right)$ when the visibility is equal to 1 .

The role of spatial information becomes more pronounced for finite visibilities $\mathcal{V}$, for which the spatial information always provides an advantage for all values of $\varepsilon$ compared to the case when we consider only the ratio of cross-coincidences and double events where the precision reads $\mathcal{V}^{2}\left[32\left(1-\mathcal{V}^{2}\right)\right]^{-1} \varepsilon^{2}+O\left(\varepsilon^{4}\right)$. This is achieved as coincidences that arise due to finite visibility are characterized by a different spatial distribution than coincidences that are due to spatial separation. In both cases we recover the $\varepsilon^{2}$ scaling, and thus for small $\varepsilon$ the advantage of the collective schemes over DI takes the form of a constant factor rather than favorable scaling. Nonetheless, as this factor scales as $(1-\mathcal{V})^{-1}$, the enhancement can be significant.

For a proof-of-principle experimental demonstration, we generated families of states $\rho^{\otimes 2}$ for a set of separations $\varepsilon$ [see Fig. 2(a) and Supplemental Material for details of the interferometric setup [41]]. In Figs. 2(c) and 2(d) we plot the final precision of estimation divided by the total number of photons used as a function of $\varepsilon$ (see Supplemental Material for details of the data analysis [41]). The proposed theory (for $\mathcal{V}=0.92$ ) accurately predicts the estimation precision for the given experimental parameters demonstrating a significant, over twofold, enhancement over the DI scheme. The spatial resolution provides an advantage over the whole range of parameters, as it allows us to distinguish effects of finite visibility versus the reduced mode overlap due to source separation.

In Figs. 2(c) and 2(d) we additionally plot the theoretical predictions for $\mathcal{V}=0.99$ and perfect interference, i.e., $\mathcal{V}=1$. The precision approaches a constant value for $\varepsilon \rightarrow 0$ only for $\mathcal{V}=1$, but offers significant enhancement for realistic visibilities. Note that if information is drawn only from the number of coincidences to double events with no spatial resolution, we can still beat the DI scheme over a broad range of parameters, especially for small $\varepsilon$. This highlights the possibility to perform precise imaging with only single-pixel detectors.

Let us now provide a simple argument for the observed degree of precision enhancement. The approximately twofold reduction of precision for the $\varepsilon$ estimation for $\mathcal{V}=1$ in the $2 \mathrm{P}$ protocol compared to the fundamental bound is due to the fact that the protocol performs collective measurement on two photons only. The essence of the collective measurement is effective projection of $\rho^{\otimes 2}$ on symmetric and antisymmetric subspaces thanks to the properties of the Hong-Ou-Mandel interference. Such a measurement commutes with joint unitary transformation of the state $U^{\otimes 2} \rho^{\otimes 2} U^{\dagger \otimes 2}$, which represents the shift of the centroid $x_{0}$ in our model, and hence is compatible with the measurement optimal for extracting information on $x_{0}$. Theoretically, if collective measurements on an arbitrary number of copies were possible, one could project the $\rho^{\otimes N}$ state on subspaces corresponding to different irreducible representation of the permutation group which provides optimal information about the $\varepsilon$ parameter in the $N \rightarrow \infty$ limit and does not interfere with the optimal measurement of $x_{0}$ [21]. Thus, through harnessing more than two photons one would be able to approach and even saturate the quantum Cramér-Rao bound Eq. (4).

Interestingly, in a slightly modified imaging scenario, the two-photon measurements may actually saturate the limit discussed above. Consider a different variant of the two-photon state impinging on the beam splitter: 

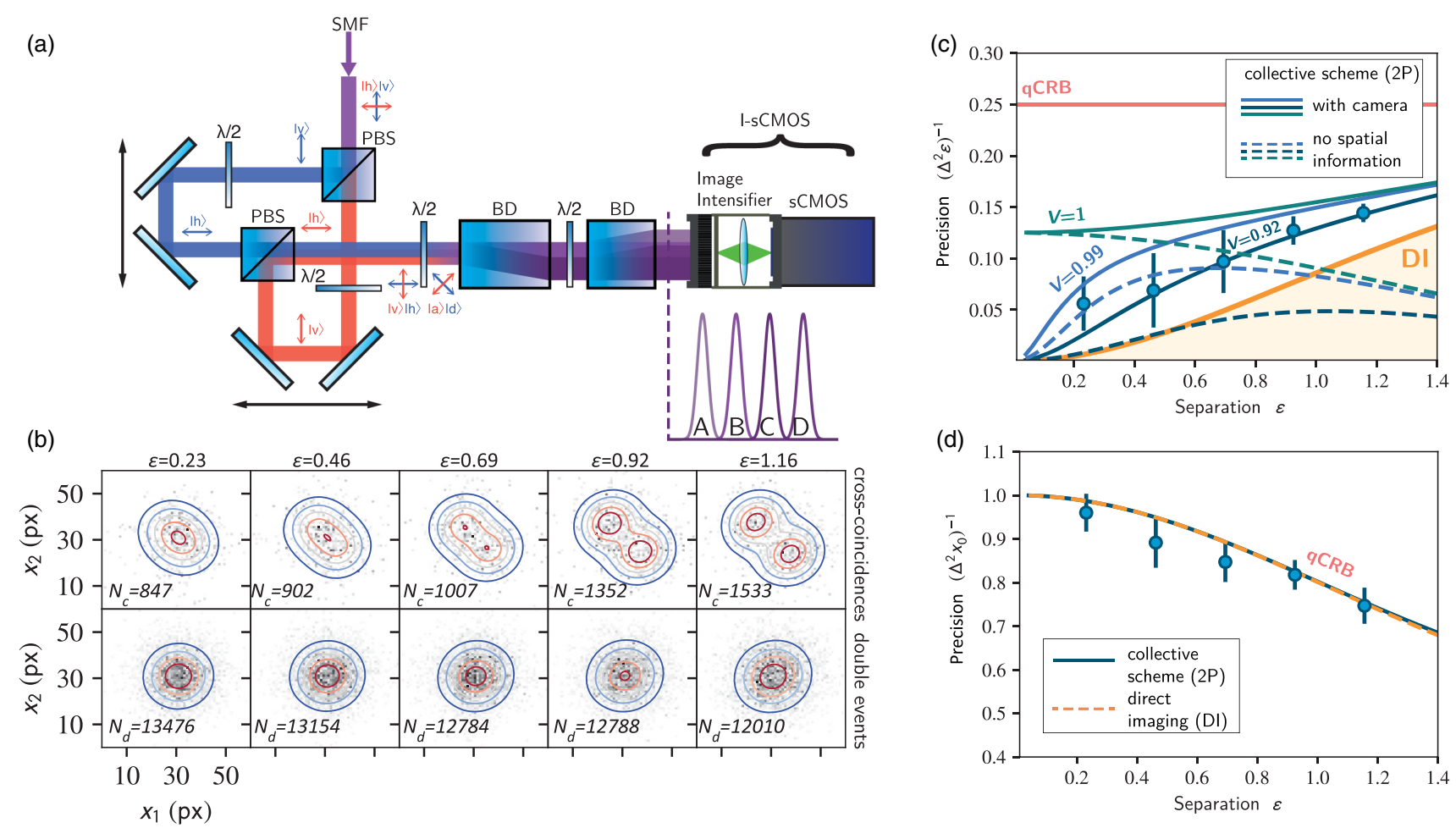

FIG. 2. Results of the multiparameter quantum estimation in a superresolution imaging scenario with a photon-pair source. (a) Experimental setup for generating a pair of photons in two adjacent modes (PBS, polarizing beam splitter; $\lambda / 2$, half-wave plate; $\mathrm{BD}$, calcite beam displacer). Using an $|h v\rangle$ photon pair and reconfiguring the positions of the retroreflectors in the interferometer, we generate the two-photon state expected in the imaging experiment for a set of values of source separation $\varepsilon$. The output single mode fiber (SMF) face is imaged onto the I-sCMOS sensor (see Supplemental Material [41] or Ref. [43] for details of I-sCMOS sensor operation and construction) photocathode so that the beam has a flat wave front with $1 / e^{2}$ diameter of $100 \mu \mathrm{m}$. The camera registers cross-coincidences (as coincidences between regions $\mathrm{A}-\mathrm{C}, \mathrm{A}-\mathrm{D}, \mathrm{B}-\mathrm{C}$, and $\mathrm{B}-\mathrm{D}$ ) and double events (as coincidences between regions $\mathrm{A}-\mathrm{B}$ or $\mathrm{C}-\mathrm{D}$ ). (b) Spatially resolved cross-coincidences (top) and double events (bottom) along with fitted model with Gaussian mode shape for subsequent values of $\varepsilon$ corresponding to data points in (c) and (d). Color scale for each map is normalized separately to highlight shape intricacies. (c) Precision of estimation of $\varepsilon$ for the $\rho^{\otimes 2}$ state and (d) precision of estimation of the centroid position $x_{0}$ as a function of source separation $\varepsilon$. The collective $2 \mathrm{P}$ scheme provides an enhancement in estimation of $\varepsilon$ while preserving the precision of centroid estimation. The ultimate precision limit given by the quantum Cramér-Rao bound is denoted by qCRB, and for the precision of centroid estimation in (d) it overlaps with the precision obtained with the protocols we employ. Theoretical curves are obtained from numerically evaluated FI. Error bars correspond to 1 standard deviation of results obtained from each data set containing 1000 coincidences (see Supplemental Material for details [41]).

$\rho_{11}=\frac{1}{2}\left(\left|x_{+}\right\rangle\left\langle x_{+}|\otimes| x_{-}\right\rangle\left\langle x_{-}|+| x_{-}\right\rangle\left\langle x_{-}|\otimes| x_{+}\right\rangle\left\langle x_{+}\right|\right)$,

which represents a situation where the photons from the two sources always enter at different input ports of the beam splitter. Such a two-photon state could be obtained from a pair of single-photon emitters excited simultaneously, where we would never observe two photons emitted from the same source. In this case, analogous calculations to the ones presented in Ref. [1] for the $\rho$ state lead to the quantum FI matrix, which in the leading order in $\varepsilon$ remains unchanged, whereas the two-photon experiment described above saturates the bound exactly:

$$
\left(\Delta^{2} x_{0}\right)_{2 \mathrm{P}, \rho_{11}}^{-1}=\left(\Delta^{2} x_{0}\right)_{Q, \rho_{11}}^{-1}=1
$$

$$
\left(\Delta^{2} \varepsilon\right)_{2 \mathrm{P}, \rho_{11}}^{-1}=\left(\Delta^{2} \varepsilon\right)_{Q, \rho_{11}}^{-1}=\frac{1}{4}
$$

Finally, it is insightful to juxtapose the presented scheme with the celebrated Hanbury Brown-Twiss (HBT) interferometry [44-48]. The essential difference is that in our approach photon positions are measured in the image plane, while in the HBT scenario spatially resolved detection is implemented in the Fourier plane conjugate to the source. For photons arriving from point sources located at angular positions specified by wave vectors $\mathbf{k}_{1}$ and $\mathbf{k}_{2}$ and detectors placed at $\mathbf{r}_{1}$ and $\mathbf{r}_{2}$, HBT interference produces fringes whose spatial variation is proportional to the expression $\cos ^{2}\left[\left(\mathbf{k}_{1}-\mathbf{k}_{2}\right)\left(\mathbf{r}_{1}-\mathbf{r}_{2}\right) / 2\right]$ [49]. If the maximum distance $\left|\mathbf{r}_{1}-\mathbf{r}_{2}\right|$, which can be viewed as the aperture of the 
measuring system, is fixed, an attempt to retrieve the angular separation between the sources from HBT fringes will suffer from the Rayleigh curse in the limit $\left|\mathbf{k}_{1}-\mathbf{k}_{2}\right| \rightarrow 0$. This is because for vanishing $\left|\mathbf{k}_{1}-\mathbf{k}_{2}\right|$ one will observe only a small fraction of the HBT fringe in the vicinity of its maximum.

In the case of the two-photon scheme presented here, we should emphasize the role of the prior QND measurement if superresolution is to be achieved with classical thermal light sources. While HBT interferometry works also with classical light sources, albeit with reduced visibility, the enhancement offered by our scheme stems from realizing two-photon interferometry sufficiently close to the dark fringe, i.e., with high visibility $\mathcal{V}$. In fact, since classical light sources can attain at most $50 \%$ visibility of Hong-OuMandel interference, Eq. (6) indicates that no significant improvement is possible over the DI scheme: for $\mathcal{V}=0.5$, we get $\left(\Delta^{2} \varepsilon\right)_{2 \mathrm{P}}^{-1}=\left(5 \varepsilon^{2} / 32\right)$ versus $\left(\Delta^{2} \varepsilon\right)_{\mathrm{DI}}^{-1}=\left(\varepsilon^{2} / 8\right)$ in the case of direct imaging.

In conclusion, we have demonstrated both theoretically and experimentally an imaging protocol that circumvents the difficulties in a multiparameter estimation problem by use of a collective measurement. The presented experimental results conclusively confirm the possibility to exploit the inherent indistinguishability of photons to perform quantum-enhanced simultaneous estimation of source separation and centroid. With this proof-of-principle experiment we have also proposed a set of realistic schemes in which our protocol could be readily applied, even to gain additional information along the traditional single-photon DI scenario or other superresolution techniques. The general theory of superresolved imaging $[1,4,7]$ implies that the same protocol might be directly applied in the case of a more general light source distribution provided one would be interested in estimating its first and second moments.

This work has been funded by the National Science Centre (Poland) Projects No. 2017/25/N/ST2/01163 and No. 2016/22/E/ST2/00559, and by the project "Quantum Optical Communication Systems" carried out within the TEAM program of the Foundation for Polish Science cofinanced by the European Union under the European Regional Development Fund. M. P. thanks M. Jachura for know-how transfer on the photon-pair source and M. Mazelanik for insightful discussions.

*michal.parniak@fuw.edu.pl

[1] J. Řehaček, Z. Hradil, B. Stoklasa, M. Paúr, J. Grover, A. Krzic, and L. L. Sánchez-Soto, Phys. Rev. A 96, 062107 (2017).

[2] M. Paúr, B. Stoklasa, Z. Hradil, L. L. Sánchez-Soto, and J. Rehacek, Optica 3, 1144 (2016).

[3] F. Yang, A. Taschilina, E. S. Moiseev, C. Simon, and A. I. Lvovsky, Optica 3, 1148 (2016).
[4] A. Chrostowski, R. Demkowicz-Dobrzański, M. Jarzyna, and K. Banaszek, Int. J. Quantum. Inform. 15, 1740005 (2017).

[5] S. Ragy, M. Jarzyna, and R. Demkowicz-Dobrzański, Phys. Rev. A 94, 052108 (2016).

[6] C. Napoli, T. Tufarelli, S. Piano, R. K. Leach, and G. Adesso, arXiv:1805.04116.

[7] S. Zhou and L. Jiang, arXiv:1801.02917.

[8] M. Tsang, Phys. Rev. Lett. 102, 253601 (2009).

[9] M. Tsang, Optica 2, 646 (2015).

[10] M. Tsang, R. Nair, and X. M. Lu, Phys. Rev. X 6, 031033 (2016).

[11] M. Tsang, New J. Phys. 19, 023054 (2017).

[12] R. Kerviche, S. Guha, and A. Ashok, arXiv:1701.04913.

[13] Z. Dutton, R. Kerviche, A. Ashok, and S. Guha, arXiv: 1801.06602 .

[14] K. G. Puschmann and F. Kneer, Astron. Astrophys. 436, 373 (2005).

[15] Z. S. Tang, K. Durak, and A. Ling, Opt. Express 24, 22004 (2016).

[16] R. Nair and M. Tsang, Phys. Rev. Lett. 117, 190801 (2016).

[17] R. Nair and M. Tsang, Astrophys. J. 808, 125 (2015).

[18] C. Lupo and S. Pirandola, Phys. Rev. Lett. 117, 190802 (2016).

[19] W.-K. Tham, H. Ferretti, and A. M. Steinberg, Phys. Rev. Lett. 118, 070801 (2017).

[20] W. E. Moerner, Rev. Mod. Phys. 87, 1183 (2015).

[21] E. Bagan, M. A. Ballester, R. D. Gill, A. Monras, and R. Muñoz-Tapia, Phys. Rev. A 73, 032301 (2006).

[22] M. D. Vidrighin, G. Donati, M. G. Genoni, X.-M. Jin, W. S. Kolthammer, M. S. Kim, A. Datta, M. Barbieri, and I. A. Walmsley, Nat. Commun. 5, 3532 (2014).

[23] M. Jachura, R. Chrapkiewicz, R. Demkowicz-Dobrzański, W. Wasilewski, and K. Banaszek, Nat. Commun. 7, 11411 (2016).

[24] L. A. Rozema, J. D. Bateman, D. H. Mahler, R. Okamoto, A. Feizpour, A. Hayat, and A. M. Steinberg, Phys. Rev. Lett. 112, 223602 (2014).

[25] Z. Liao, M. Al-Amri, and M. Suhail Zubairy, Phys. Rev. Lett. 105, 183601 (2010).

[26] R. W. Boyd, J. P. Dowling, and R. W. Boyd, Quantum Inf. Process. 11, 891 (2012).

[27] M. D’Angelo, M. V. Chekhova, and Y. Shih, Phys. Rev. Lett. 87, 013602 (2001).

[28] A. N. Boto, P. Kok, D. S. Abrams, S. L. Braunstein, C. P. Williams, and J. P. Dowling, Phys. Rev. Lett. 85, 2733 (2000)

[29] O. Firstenberg, T. Peyronel, Q.-Y. Liang, A. V. Gorshkov, M. D. Lukin, and V. Vuletić, Nature (London) 502, 71 (2013).

[30] M. Parniak, M. Dąbrowski, M. Mazelanik, A. Leszczyński, M. Lipka, and W. Wasilewski, Nat. Commun. 8, 2140 (2017).

[31] M. Parniak, M. Mazelanik, A. Leszczyński, M. Lipka, M. Dąbrowski, and W. Wasilewski, arXiv:1804.05854.

[32] D. Tiarks, S. Schmidt, G. Rempe, and S. Dürr, Sci. Adv. 2, e1600036 (2016).

[33] H. Busche, P. Huillery, S. W. Ball, T. Ilieva, M. P. A. Jones, and C. S. Adams, Nat. Phys. 13, 655 (2017). 
[34] E. Distante, P. Farrera, A. Padrón-Brito, D. Paredes-Barato, G. Heinze, and H. de Riedmatten, Nat. Commun. 8, 14072 (2017).

[35] L. Li and A. Kuzmich, Nat. Commun. 7, 13618 (2016).

[36] J. L. Everett, G. T. Campbell, Y.-W. Cho, P. Vernaz-Gris, D. Higginbottom, O. Pinel, N. P. Robins, P. K. Lam, and B. C. Buchler, Nat. Phys. 13, 68 (2016).

[37] T.-S. Yang, Z.-Q. Zhou, Y.-L. Hua, X. Liu, Z.-F. Li, P.-Y. Li, Y. Ma, C. Liu, P.-J. Liang, X. Li, Y.-X. Xiao, J. Hu, C.-F. Li, and G.-C. Guo, Nat. Commun. 9, 3407 (2018).

[38] V. Walther, R. Johne, and T. Pohl, Nat. Commun. 9, 1309 (2018).

[39] S. M. Kay, Fundamentals of Statistical Signal Processing: Estimation Theory, edited by S. E. A. V. Oppenheim (Prentice-Hall, Englewood Cliffs, NJ, 1993).

[40] C. W. Helstrom, Quantum Detection and Estimation Theory (Academic Press, New York, 1976).

[41] See Supplemental Material at http://link.aps.org/ supplemental/10.1103/PhysRevLett.121.250503 for details of derivations, photon-pair source operation, spatiallyresolved detection, and data analysis, which includes Ref. [42].

[42] M. Lipka, M. Parniak, and W. Wasilewski, Appl. Phys. Lett. 112, 211105 (2018).

[43] R. Chrapkiewicz, M. Jachura, K. Banaszek, and W. Wasilewski, Nat. Photonics 10, 576 (2016).

[44] R. Hanbury Brown and R. Q. Twiss, Philos. Mag. 45, 633 (1954).

[45] R. Hanbury Brown and R. Q. Twiss, Nature (London) 178, 1046 (1956).

[46] R. Hanbury Brown and R. Q. Twiss, Proc. R. Soc. A 242, 300 (1957).

[47] R. Hanbury Brown and R. Q. Twiss, Proc. R. Soc. A 243, 291 (1958).

[48] U. Fano, Am. J. Phys. 29, 539 (1961).

[49] M. O. Scully and M.S. Zubairy, Quantum Optics (Cambridge University Press, Cambridge, England, 1997). 